\title{
The temperature effect on electrokinetic properties of the silica-polyvinyl alcohol (PVA) system
}

\author{
Małgorzata Wiśniewska
}

Received: 6 September 2010 /Revised: 27 October 2010 / Accepted: 12 November 2010 /Published online: 1 December 2010

(C) The Author(s) 2010. This article is published with open access at Springerlink.com

\begin{abstract}
The influence of polyvinyl alcohol (PVA) adsorption on the structure of the diffuse layer of silica $\left(\mathrm{SiO}_{2}\right)$ in the temperature range $15-35{ }^{\circ} \mathrm{C}$ was examined. The microelectrophoresis method was used in the experiments to determine the zeta potential of the solid particles in the absence and presence of the polymer. The adsorption of PVA macromolecules causes the zeta potential decrease in all investigated $\mathrm{SiO}_{2}$ systems. Moreover this, decrease is the most pronounced at the highest examined temperature. Obtained results indicate that the conformational changes of adsorbed polymer chains are responsible for changes in electrokinetic properties of silica particles. Moreover, the structure of diffuse layer on the solid surface with adsorbed polymer results from the following effects: the presence of acetate groups in PVA chains, the blockade of silica surface groups by adsorbed polymer and the shift of slipping plane due to macromolecules adsorption.
\end{abstract}

Keywords Polyvinyl alcohol $\cdot$ Silica $\cdot$ Polymer conformation $\cdot$ Zeta potential $\cdot$ Slipping plane $\cdot$ Temperature influence

\section{Introduction}

The addition of macromolecular substance to colloidal system is a very good way to change of its stabilization-flocculation conditions. These properties of polymers result from the possibility of their macromolecules to adsorb at the interfaces

\footnotetext{
M. Wiśniewska $(\bowtie)$

Department of Radiochemistry and Colloids Chemistry, Faculty of Chemistry, Maria Curie Sklodowska University, M. Curie Sklodowska Sq. 3, 20-031 Lublin, Poland

e-mail: wisniewska@hektor.umcs.lublin.pl
}

with wide variety of different conformations. But, not only adsorbed macromolecules determine the stability of the solid suspension (steric stabilization, bridging flocculation). In the range of high polymer concentrations, the unadsorbed macromolecules, freely dispersed in the solution, can cause depletion interactions. These forces result in depletion stabilization or depletion flocculation occurrence.

The stabilization properties show usually polymeric dispersants with molecular weight less than $20,000 \mathrm{~g} /$ mole [1] The optimum thickness of dispersant adsorption layer has to be sufficient to overcome the van der Waals attraction between colloidal particles. The stabilization process in the presence of polymer is widely used in production of cosmetics, paints, coatings, and pharmaceuticals as well as in food processing. On the other hand, the most efficient flocculants are the polymer with molecular weight above $1,000,000 \mathrm{~g} / \mathrm{mole}$. Such long polymer chains can absorb on two particles simultaneously (or their greater number) causing particles bridging and flocculation. This destabilization effect finds the broad application in environment protection (water purification, separation of hazardous solids from chemical waste, oil recovery), decrease of ploughlandserosion, flotation as well as paper and mineral technology (purification of paper pulp and ceramic powders).

The polymer (type, concentration, polydispersity, and molecular weight) and adsorbent characteristics as well as solution conditions ( $\mathrm{pH}$, ionic strength, temperature) are the most important factors which influence the conformation of polymer chains [2]. From among these factors, the temperature effect on adsorption mechanism of polymer is scarcely and not extensively discussed in the world literature [3, 4]. Nevertheless, the stabilization-flocculation properties of colloidal suspension can be controlled by temperature changes [5]. The transition from stable to unstable region in given adsorbent-polymer system can be realized by its heating or freezing. 
Taking the above into consideration, the aim of present manuscript is to determine the electrokinetic properties of silica $\left(\mathrm{SiO}_{2}\right)$ surface in the presence of polyvinyl alcohol (PVA) at different temperatures. The zeta potential results, presented in this paper, are continuation and supplement of my previous investigations concerning the electric characteristic of $\mathrm{SiO}_{2}-\mathrm{PVA}$ systems within $15-35{ }^{\circ} \mathrm{C}$ temperature range [6]. The knowledge about structure of the polymer adsorption layer at different temperatures can be very helpful to determine the macromolecule conformation changes. This fact is very important for many technological processes which take place at different temperatures to obtain the required stabilization or destabilization effects.

\section{Materials and methods}

In the experiments, the samples used were of silica- $\mathrm{SiO}_{2}$ (Merck). The solid was washed with doubly distilled water to eliminate the inorganic contamination. The supernatant conductivity after washing process was lower than $2 \mu \mathrm{S} / \mathrm{cm}$. The BET surface area of silica was $326 \mathrm{~m}^{2} / \mathrm{g}$. It was determined using the automatic adsorption analyzer ASAP 2405 (Micromeritics Instrument) from the low-temperature nitrogen adsorption-desorption method.

The samples of polyvinyl alcohol-PVA (Fluka) with two weight average molecular weights 22,000 and 38,000 were used in the study. The degree of their hydrolysis was $97.5 \%$. Prepared polymer solutions were filtered through the cellulose membranes (Millipore). Such process leads to eliminate inorganic contamination and minimalize the range of the molecular weights in polymer samples.

Despite the fact, that PVA is classified as a nonionic polymer, its macromolecules contain besides hydroxyl groups $(-\mathrm{OH})$ some acetate ones $\left(-\mathrm{OCOCH}_{3}\right)$. These groups come from uncompleted hydrolysis of polyvinyl acetate in the production process of PVA, according to reactions:
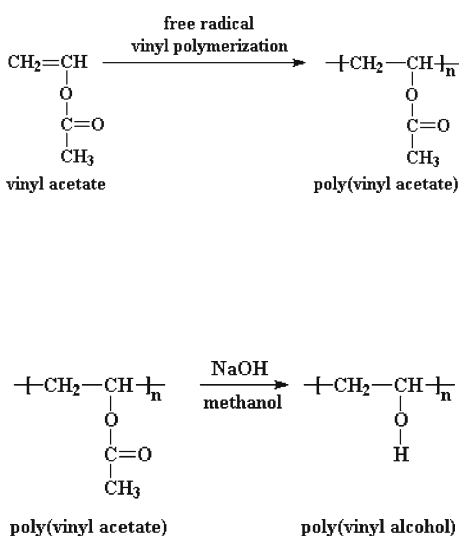

The polyvinyl alcohol used in the experiments was characterized by degree of hydrolysis $97.5 \%$. Thus, its macromolecules contain $2.5 \%$ acetate groups. The polyvinyl alcohol chains have the following structure:

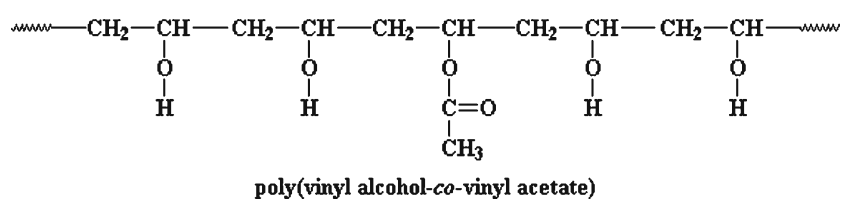

The $\mathrm{C}-\mathrm{H}$ bonds in $\alpha$ position in relation to acetate groups have acidic properties. In this way the acetate groups in PVA macromolecules gain negative charge. The ionization of acetate groups in PVA chains are illustrated by the hollowing reaction:

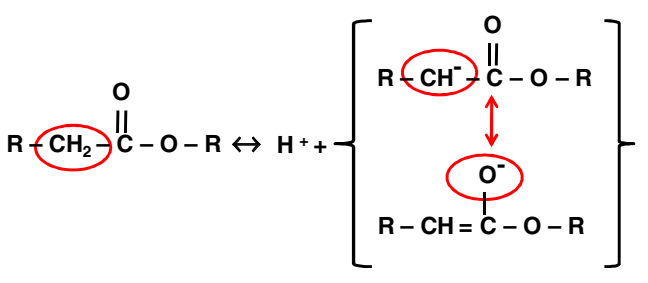

The silica samples for zeta potential measurements were prepared adding $0.03 \mathrm{~g}$ of $\mathrm{SiO}_{2}$ to $300 \mathrm{~cm}^{3}$ of the supporting electrolyte solution $\left(\mathrm{C}_{\mathrm{NaCl}}=1 \times 10^{-2} \mathrm{~mole} / \mathrm{dm}^{3}\right)$. This suspension was sonicated (ultrasonic processor $\mathrm{XL}$, Misonix) for $5 \mathrm{~min}$ and divided into five parts of $60-\mathrm{cm}^{3}$ volume. Next, the appropriate $\mathrm{pH}$ value (ranging from 3 to 10) was adjusted in each sample. All samples were termostated at the time of their preparation. In the similar way were prepared silica systems in the presence of polyvinyl alcohol. For this purpose the appropriate volume of polymer with initial concentration $1,250 \mathrm{ppm}$ was added to supporting electrolyte solution, which led to its final concentration $C_{\mathrm{PVA}}=100 \mathrm{ppm}$. The further way of probes preparation was the same as in the case of $\mathrm{SiO}_{2}$ without PVA.

The zeta potentials of the silica particles in the presence and absence of polyvinyl alcohol were measured with the Zetasizer 3,000 laser zetameter (Malvern Instruments).

All zeta potential measurements were carried out in the temperature range $15-35{ }^{\circ} \mathrm{C}$.

\section{Results}

The analysis of obtained curves (Figs. 1, 2, and 3) indicates that at all investigated temperatures the zeta potential of 


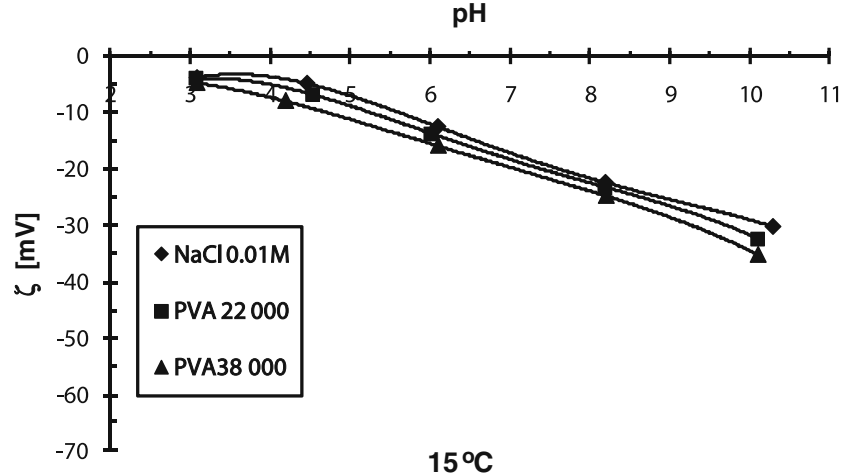

Fig. 1 Zeta potential of $\mathrm{SiO}_{2}$ particles in the absence and presence of PVA at $15^{\circ} \mathrm{C} ; C_{\mathrm{PVA}}=100 \mathrm{ppm}, C_{\mathrm{NaCl}}=1 \times 10^{-2} \mathrm{~mol} / \mathrm{dm}^{3}$

$\mathrm{SiO}_{2}$ in the absence of PVA is negative in the whole range of studied $\mathrm{pH}$. The adsorption of polyvinyl alcohol causes the decrease of the diffuse layer charge of the silica. The higher the temperature is, the more pronounced reduction in zeta potential of solid suspension is observed.

\section{Discussion}

The explanation of the obtained dependencies requires the analysis of changes in macromolecules ionization and solid surface charge density as a function of solution $\mathrm{pH}$. The concentration of various types of silica surface groups at $25{ }^{\circ} \mathrm{C}$ are presented in Table 1 . These data are obtained by numerical optimalization of the electrical three layer model, using dependence of surface charge density versus $\mathrm{pH}$. Practically in the whole range of studied $\mathrm{pH}$, the electrostatic repulsion between the negatively charged surface $\left(\mathrm{pH}_{\mathrm{pzc}} \approx 3-4[6,7]\right)$ and ionized acetate groups in PVA macromolecules occurs. For this reason, adsorption of ionized PVA chains on the negatively charged silica surface undergoes through hydrogen bridges interaction. The hydrogen bonds can be created between silanol groups (both neutral $(-\mathrm{SiOH})$ and charged $\left.\left(-\mathrm{SiO}^{-},-\mathrm{SiOH}_{2}{ }^{+}\right)\right)$and polymer functional

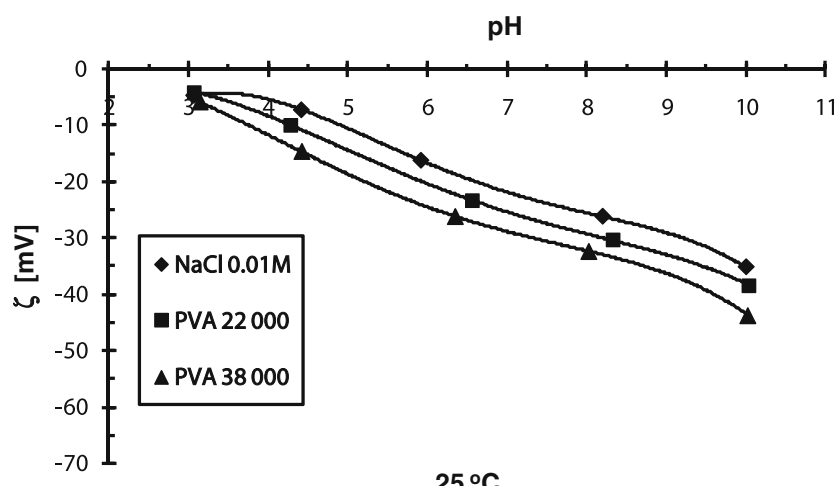

Fig. 2 Zeta potential of $\mathrm{SiO}_{2}$ particles in the absence and presence of PVA at $25^{\circ} \mathrm{C}, C_{\mathrm{PVA}}=100 \mathrm{ppm}, C_{\mathrm{NaCl}}=1 \times 10^{-2} \mathrm{~mol} / \mathrm{dm}^{3}$

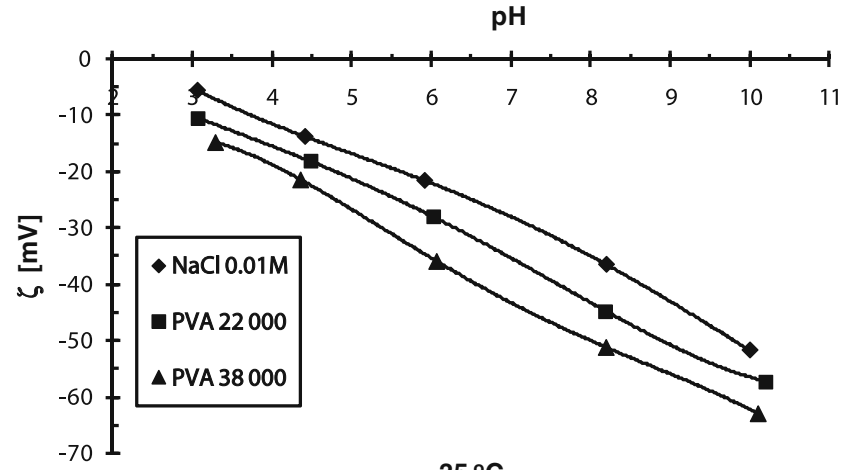

$35^{\circ} \mathrm{C}$

Fig. 3 Zeta potential of $\mathrm{SiO}_{2}$ particles in the absence and presence of PVA at $35^{\circ} \mathrm{C}, C_{\mathrm{PVA}}=100 \mathrm{ppm}, C_{\mathrm{NaCl}}=1 \times 10^{-2} \mathrm{~mol} / \mathrm{dm}^{3}$

groups $\left(-\mathrm{OH},-\mathrm{OCOCH}_{3}\right.$, and $\left.-\mathrm{OCO}^{-} \mathrm{CH}_{3}\right)$. Previous investigations also indicated that acetate groups show preferential adsorption on the metal oxide surface in comparison to hydroxyl ones [8].

The obtained lowering of zeta potential of silica particles in the presence of polymer can be caused by three effects: the shift of slipping plane due to polymer adsorption, the blockade of solid active sites by the previously adsorbed polymer chains and the presence of functional groups in macromolecules [9].

The anionic character of PVA chains, containing ionized acetate groups, has some influence on reduction of zeta potential in whole investigated temperature range. These negative-charged groups are present in the interface area with tail and loops structures of adsorbed polymer chains and influence the diffuse layer charge.

The temperature rise causes more distinct decrease of the silica zeta potential in the presence of polyvinyl alcohol (Figs. 1, 2, and 3). Such behavior of investigated systems results from the changes of linear dimension of adsorbing macromolecules with increasing temperature. The previous studies indicated that values of parameters characterizing conformation of polymer chains in the solution $\left(\alpha_{\exp }\right.$ expansion coefficient, $\left(\mathrm{r}^{-2}\right)^{1 / 2}$-root-mean-square chain end-to-end distance, $\mathrm{R}_{\mathrm{h}}$ - hydrodynamic radius of a polymer coil) increase with rising temperature [10]. It means that macromolecules become more stretched structure.

Table 1 Concentration of various types of silica surface groups in the presence of $\mathrm{NaCl}\left(C_{\mathrm{NaCl}}=1 \times 10^{-2} \mathrm{~mol} / \mathrm{dm}^{3}\right)$ at $25^{\circ} \mathrm{C}$

\begin{tabular}{lccc}
\hline $\mathrm{pH}$ & $\mathrm{SiOH}_{2}{ }^{+}\left[\mu \mathrm{C} / \mathrm{cm}^{2}\right]$ & $\mathrm{SiO}^{-}\left[\mu \mathrm{C} / \mathrm{cm}^{2}\right]$ & $\mathrm{SiOH}\left[\mu \mathrm{C} / \mathrm{cm}^{2}\right]$ \\
\hline 4 & 0.001 & 0.000 & 135.306 \\
5 & 0.000 & -0.004 & 135.305 \\
6 & 0.000 & -0.061 & 135.247 \\
7 & 0.000 & -0.400 & 134.898 \\
8 & 0.000 & -2.333 & 132.914 \\
9 & 0.000 & -7.717 & 126.863 \\
\hline
\end{tabular}


Taking the above into consideration, the conformation of adsorbed PVA chains is more coiled at low examined temperature $\left(15^{\circ} \mathrm{C}\right)$. Such structure of adsorption layers consisted with polymer coils causes the effect of blockade of the solid active sites. These sites are inaccessible for electrolyte ions and other polymer chains, resulting in changes in diffuse layer composition. Thus, at these temperature conditions the effect of blockade of the solids surface sites is mainly responsible for the changes of zeta potential of $\mathrm{SiO}_{2}$ particles in the presence of polymer. Additionally, more coiled structure of adsorbed chains causes closing of great number of negatively charged acetate groups inside those coils. For this reason, the small number of negatively charged acetate groups is present in the diffuse layer area and slightly influence the zeta potential of silica particles.

When temperature rises, polymer coils develop and the effect of the slipping plane shift start to be dominant. In this situation, adsorbed on the solid surface polymer chains creating more stretched conformation with numerous loop and tail structures. As a consequence, the greater shift of slipping plane and more pronounced reduction of the zeta potential are observed. Moreover, the stretched conformation of adsorbed macromolecules ensures that a greater number of acetate groups are located in diffuse layer. The negative charge originating from these groups can have some contribution to zeta potential lowering at higher investigated temperatures.

The extent of the screening of solid surface charge by the PVA macromolecules depends on polymer chain conformation. This conformation becomes more stretched with the $\mathrm{pH}$ increase and temperature rise. The zeta potential changes reflect this effect. The more stretched conformation of polymer chains is, the weaker screening of surface groups should be observed. In this situation a great number of acetate groups are present in the slipping plane area causing more pronounced reduction of zeta potential. Thus, the lower the reduction of zeta potential is, the greater extent of the screening of solid surface charge by the adsorbed PAA molecules is obtained.

\section{Conclusions}

The temperature changes in the range $15-35^{\circ} \mathrm{C}$ influences the silica zeta potential in the presence of polyvinyl alcohol.
The polymer adsorption causes decrease of the diffuse layer charge at all investigated temperatures. The higher the temperature is, the greater lowering in zeta potential is observed. The main reason for reduction of the silica zeta potential with adsorbed PVA is presence of ionized acetate groups in these chains. Additionally, the effect of blockade of the solid active sites by the adsorbed polymer coils is mainly responsible for electrokinetic behavior of the investigated systems at $15{ }^{\circ} \mathrm{C}$. At $35{ }^{\circ} \mathrm{C}$ the shift of slipping plane due to adsorption of stretched polymer chains, is dominant.

Open Access This article is distributed under the terms of the Creative Commons Attribution Noncommercial License which permits any noncommercial use, distribution, and reproduction in any medium, provided the original author(s) and source are credited.

\section{References}

1. Farrokhpay S (2009) A review of polymeric dispersant stabilization of titania pigment. Adv Colloid Interface Sci 151:24-32

2. Tadros T (2009) Polymeric surfactants in disperse systems. Adv Colloid Interface Sci 147-148:281-299

3. Guo LC, Zhang Y, Uchida N, Uematsu K (1997) Influence of temperature on stability of aqueous alumina slurry containing polyelectrolyte dispersant. J Eur Ceram Soc 17:345-350

4. Mpofu P, Addai-Mensah J, Ralston J (2004) Temperature influence of nonionic polyethylene oxide and anionic polyacrylamide on flocculation and dewatering behaviour of kaoline dispersions. J Colloid Interface Sci 271:145-156

5. Napper DH (1983) Polymeric stabilization of colloidal dispersions. Academic, London

6. Wiśniewska M (2010) The structure of electrical double layer of silica in the presence of polyvinyl alcohol (PVA) at different temperatures. Mat Let 64:1611-1613

7. Liufu SC, Xiao HN, Li YP (2005) Adsorption of cationic polyelectrolyte at the solid/liquid interface and dispersion of nanosized silica in water. J Colloid Interface Sci 285:33-40

8. Chibowski S, Paszkiewicz M, Krupa M (2000) Investigation of the influence of the polyvinyl alcohol adsorption on the electrical properties of $\mathrm{Al}_{2} \mathrm{O}_{3}$-solution interface, thickness of the adsorption layers of PVA. Powder Technol 107:251-255

9. M'Pandou A, Siffert B (1987) Polyethyleneglycol adsorption at the $\mathrm{TiO}_{2}-\mathrm{H}_{2} \mathrm{O}$ interface: distortion of ionic structure and shear plane position. Colloids Surf 24:159-172

10. Wiśniewska M (2010) Influences of polyacrylic acid adsorption and temperature on the alumina suspension stability. Powder Technol 198:258-266 